\title{
La propuesta de la Unión Europea relativa a un impuesto sobre el carbono en frontera $y$ su compatibilidad con las normas de la Organización Mundial del Comercio
}

The European Union proposal on a carbon border tax and its compatibility with the World Trade Organization rules

\author{
Xavier Fernández Pons \\ Profesor Titular de Universidad \\ Derecho Internacional Público \\ Miembro Cátedra Jean Monnet EUEL (European Union Environmental Law) \\ Universitat de Barcelona \\ E-mail: xavierfernandez@ub.edu
}

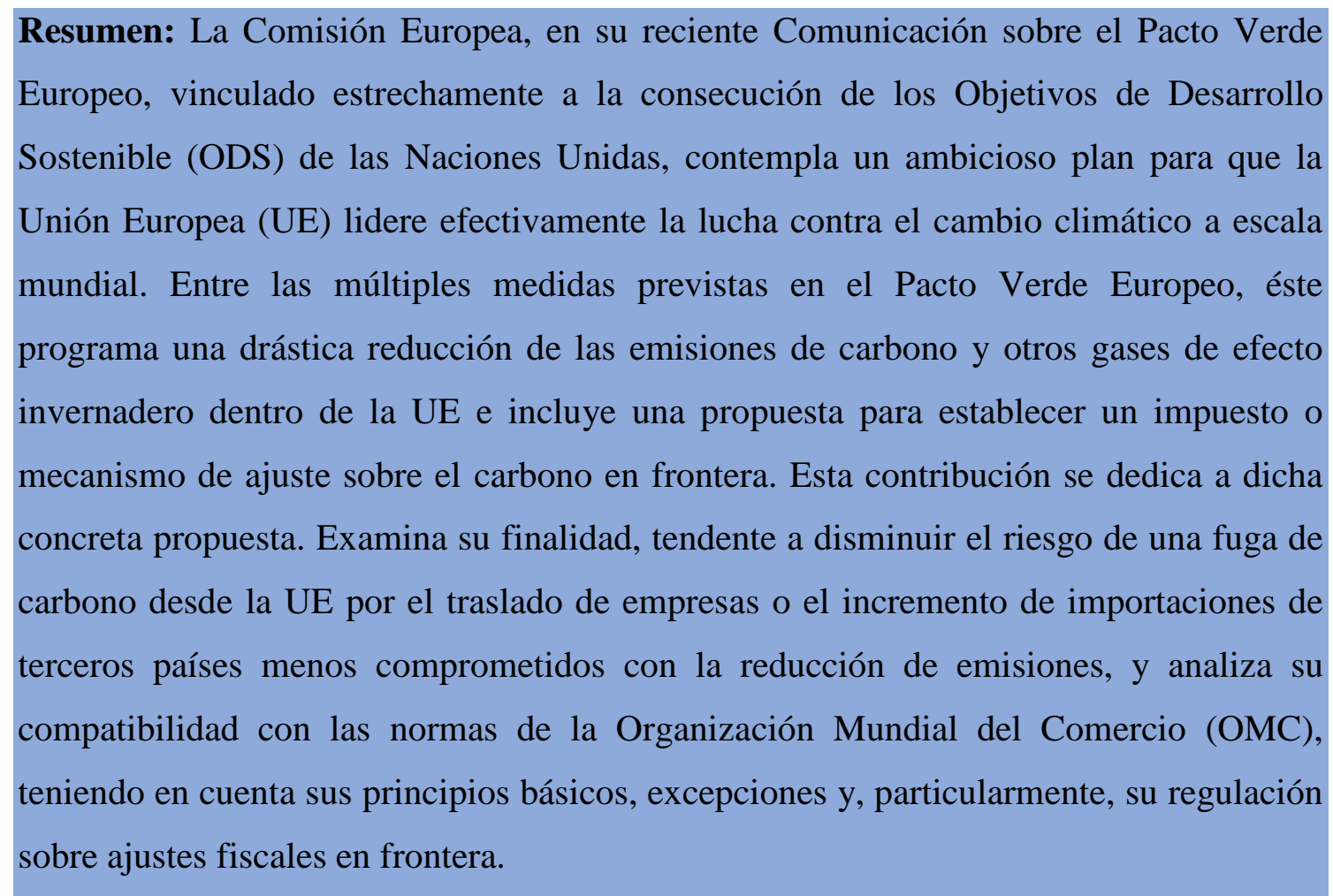




\begin{abstract}
Palabras clave: cambio climático, Objetivos de Desarrollo Sostenible, Unión Europea, fuga de carbono, impuesto sobre el carbono en frontera, ajuste fiscal en frontera, Organización Mundial del Comercio.
\end{abstract}

Abstract: The European Commission, in its recent Communication on the European Green Pact, closely linked to the achievement of the United Nations Sustainable Development Goals (SDGs), envisages an ambitious plan to make the European Union (EU) an effective global leader in the fight against climate change. Among the many measures contemplated in the European Green Pact, it programmes a drastic reduction of carbon and other greenhouse gas emissions within the EU and includes a proposal to establish a carbon border tax or carbon border adjustment. This contribution is dedicated to this specific proposal. It examines its purpose, aimed at reducing the risk of a carbon leakage from the EU due to the transfer of companies or the increase in imports from third countries less committed to reducing emissions, and it analyses their compatibility with the rules of the World Trade Organization (WTO), taking into account its basic principles, exceptions and, particularly, its regulation on border tax adjustments.

Keywords: climate change, Sustainable Development Goals, European Union, carbon leakage, carbon border tax, border tax adjustment, World Trade Organization.

\title{
1. Introducción
}

La lucha contra el cambio climático o calentamiento global, inducido por las emisiones de carbono y otros gases de efecto invernadero derivadas de la actividad humana, es uno de los principales retos para la consecución de un desarrollo sostenible ${ }^{1}$. Las Naciones Unidas, con su Agenda 2030 y los llamados ODS aprobados mediante la Resolución 70/1 de la Asamblea General de 25 de septiembre de 2015, lo corroboran. Así, se dedica específicamente el ODS 13 a la adopción de "medidas urgentes para

\footnotetext{
${ }^{1}$ Tal como vienen evidenciando los sucesivos informes del Grupo Intergubernamental de Expertos sobre el Cambio Climático de las Naciones Unidas creado en 1988 y más conocido por el acrónimo en inglés IPCC (Intergovernmental Panel on Climate Change). Por ejemplo, un reciente informe especial del IPCC (2018) se dedica, literalmente, a evaluar "los impactos del calentamiento global de $1,5^{\circ} \mathrm{C}$ con respecto a los niveles preindustriales y las trayectorias correspondientes que deberían seguir las emisiones mundiales de gases de efecto invernadero, en el contexto del reforzamiento de la respuesta mundial a la amenaza del cambio climático, el desarrollo sostenible y los esfuerzos por erradicar la pobreza".
} 
combatir el cambio climático y sus efectos", reconociendo que la Convención Marco de las Naciones Unidas sobre el Cambio Climático (con el vigente Acuerdo de París de 2015) es el principal foro intergubernamental internacional para negociar la respuesta mundial al cambio climático. A su vez, otros ODS, que siempre hay que entender como unos objetivos sociales, ambientales y económicos interconectados, también inciden, en mayor o menor medida, en este problema global (Fernández Pons, 2015).

La nueva presidenta de la Comisión Europea, Ursula von der Leyen, anunció en sus Orientaciones Políticas para 2019-2024, presentadas al Parlamento Europeo el 16 de julio de 2019, su voluntad de impulsar un "Pacto Verde Europeo", a fin de que la UE lidere la lucha contra el cambio climático a escala mundial y contribuya decisivamente a alcanzar los ODS (von der Leyen, 2019). Entre las medidas contempladas por la presidenta de la Comisión Europea, destaca la propuesta de implantar, según su tenor literal en español, un "impuesto sobre el carbono en frontera", designado en la versión en inglés como "carbon border tax" (von der Leyen, 2019: 5).

El 11 de diciembre de 2019, la Comisión Europea presentó su Comunicación titulada El Pacto Verde Europeo, que es descrito como una "nueva estrategia de crecimiento destinada a transformar la UE en una sociedad equitativa y próspera, con una economía moderna, eficiente en el uso de los recursos y competitiva, en la que no habrá emisiones netas de gases de efecto invernadero en 2050 y el crecimiento económico estará disociado del uso de los recursos" (Comisión Europea, 2019: 2). El Pacto Verde Europeo se vincula explícitamente a la integración de los ODS “en el centro de la elaboración de políticas y la acción de la UE", procurando "situar la sostenibilidad y el bienestar de los ciudadanos en el centro de la política económica" (Comisión Europea, 2019: 3).

El Pacto Verde Europeo detalla más la citada propuesta de la presidenta de la Comisión Europea sobre un "mecanismo de ajuste del carbono en frontera", que tendrá el fin de "atenuar el riesgo de fuga de carbono" y se configurará de manera que respete, entre otras obligaciones internacionales de la UE, la "normativa de la Organización Mundial del Comercio" (Comisión Europea, 2019: 6).

En un anexo de la Comunicación relativa al Pacto Verde Europeo se incluye una "hoja de ruta" legislativa, con un "calendario indicativo" de las múltiples actuaciones clave para su desarrollo, previendo la concreción de la propuesta de revisión de la Directiva sobre fiscalidad de la energía en junio de 2021 y de la propuesta de un 
"mecanismo de ajuste del carbono en frontera para sectores específicos" dentro de ese mismo año 2021 (Comisión Europea, 2019, anexo; Puig Marcó, 2020).

Aunque está por ver cómo se acabará diseñando este mecanismo de ajuste del carbono en frontera por la UE (Mehling et al., 2019; Krenek, 2020), la doctrina ya viene analizando desde hace tiempo la lógica general de tales mecanismos de ajuste o impuestos sobre el carbono en frontera (Metcalf y Weisbach, 2009; Elliott et al., 2010; Gros y Egenhofer, 2011). Dichos mecanismos consisten, de modo sumario, en gravar ciertos productos importados seleccionados por sectores, generalmente en la frontera o aduana, en función de las emisiones de carbono generadas durante su proceso de producción en origen. Así, por ejemplo, el acero importado de un país donde se produce con menores emisiones de carbono y el acero importado de otro país donde se elabora con mayores emisiones de carbono quedarían sujetos en la aduana, pese a ser productos físicamente similares o incluso idénticos, a unas "carbon border taxes" distintas, en función de su respectiva huella de carbono ${ }^{2}$.

Esta contribución se propone, en primer lugar, hacer algunas reflexiones sobre la finalidad de la indicada propuesta de la UE y, en segundo lugar, analizar las claves jurídicas que determinarían su compatibilidad con las normas de la OMC.

\section{Finalidad de la propuesta: atenuar el riesgo de fuga de carbono}

Como se ha avanzado, tanto las citadas Orientaciones Políticas de la presidenta de la Comisión Europea como la Comunicación sobre el Pacto Verde Europeo señalan que el fin a perseguir con el establecimiento de un impuesto o mecanismo de ajuste sobre el carbono en frontera es evitar o, por lo menos, atenuar el "riesgo de fuga de carbono" (von der Leyen, 2019: 5; Comisión Europea, 2019: 6). La llamada fuga del carbono es un fenómeno que ya ha sido ampliamente analizado entre la doctrina (Kuik y Gerlagh, 2003; Babiker, 2005; Peters, 2010; Arce González, 2014). Se da cuando los esfuerzos de un país en la reducción de las emisiones de carbono llevan a que ciertas industrias se trasladen a otros países, más permisivos en materia de emisiones, y/o a que se incrementen las importaciones de productos originarios de tales países menos exigentes, implicando que, globalmente, no se acaba dando una disminución de las emisiones de carbono sino su relocalización de unos países a otros. Así, de poco

\footnotetext{
2 La huella del carbono puede cuantificarse según una metodología detallada por la Organización Internacional de Normalización o Estandarización (en inglés, International Standardization Organization o ISO) que es analizada, entre otros, por García y Freire (2014), Álvarez Gallego (2015) y Wu, Xia y Wang (2015).
} 
servirán severas reducciones en las emisiones de carbono dentro de la UE si la producción que causa esas emisiones se acaba estableciendo y/o incrementando en otros países, generando desde allí las emisiones.

La Comunicación sobre el Pacto Verde Europeo contempla un ambicioso plan para que en la UE no haya emisiones netas de gases de efecto invernadero en 2050, elevando el objetivo de reducción de las emisiones de ahora al 2030 al $50 \%$, como mínimo (Comisión Europea, 2019: 5). Para lograr tan cuantiosas reducciones de emisiones, se prevé reformar diversos instrumentos de la UE contra el cambio climático, como el Régimen de Comercio de Derechos de Emisión, extendiéndolo a más sectores (Comisión Europea, 2019: 5).

Por tanto, si otros países no comparten una ambición comparable a la de la UE en los próximos años, lo cual es bastante probable teniendo en cuenta la laxitud del régimen previsto por el Acuerdo de París (Lomborg, 2015) y el desdén con el que líderes de algunas destacadas potencias han venido enfocando la cuestión del cambio climático, el riesgo de fuga de carbono podría incrementarse notablemente, frustrando los esfuerzos de la UE y perjudicando a los productores establecidos en la misma.

Hasta ahora, la UE había tratado de afrontar la fuga de carbono con diversas medidas de apoyo a los sectores de la UE más amenazados por las deslocalizaciones y la competencia exterior, como "la asignación gratuita de derechos de emisión o la compensación por la subida de los costes de la electricidad" (Comisión Europea, 2019: 6). Pero, en el nuevo escenario que plantea el Pacto Verde Europeo, tales medidas tradicionales no parece que basten y se requerirá un impuesto o mecanismo de ajuste del carbono en frontera, que grave las importaciones de productos más intensivos en la generación de emisiones de carbono y desaliente, efectivamente, el traslado de industrias desde la UE a terceros países en la búsqueda "paraísos del carbono" o “carbon havens" (Branger y Quirion, 2014: 53).

La fuga de carbono se presenta, pues, como un claro ejemplo de los problemas que, en términos más generales, pueden darse entre la protección del medio ambiente y la liberalización del comercio a escala mundial. Así, mientras algunos países se esfuerzan en elevar los estándares ambientales de sus procesos y métodos de producción, otros pueden optar por mantenerlos bajos o rebajarlos para favorecer a sus exportaciones y atraer nuevas inversiones (Eliste y Fredriksson, 2004; Woods, 2006). 
Por consiguiente, se comprende que la UE esté previendo un impuesto o mecanismo de ajuste sobre el carbono en frontera, que incida en el comercio internacional, condicionando las importaciones de ciertos productos. Ello casa plenamente, además, con la actual estrategia de la UE para impulsar un comercio internacional más sostenible y justo, que supedite las importaciones de productos y servicios al respeto de ciertas condiciones ambientales y sociales en los países de origen (Wouters et al., 2015; Douma, 2017; Gruni, 2018; Hadjiyianni, 2019).

Ahora bien, uno de los principales interrogantes jurídicos que plantea la indicada propuesta es acerca de su compatibilidad con las normas de la OMC, que vinculan internacionalmente a la UE y sus Estados miembros. En tal cuestión trata de adentrarse el siguiente apartado.

\section{Compatibilidad de la propuesta con las normas de la Organización Mundial del} Comercio (OMC)

Entre la doctrina se ha venido discutiendo mucho sobre si los impuestos o mecanismos de ajuste sobre el carbono en frontera pueden ser compatibles o no con las vigentes normas de la OMC (Sindico, 2008; Veel, 2009; Kaufmann y Weber, 2011; Pauwelyn, 2013; Gómez, 2013; Holzer, 2014; Pirlot, 2017; Trachtman, 2017a; Porterfield, 2019; Will, 2019). Los órganos de la OMC con funciones jurisdiccionales (Grupos Especiales y Órgano de Apelación) todavía no han tenido la ocasión de pronunciarse específicamente sobre esta cuestión y persisten incertidumbres jurídicas (Condon, 2009; Cottier, Nartova y Bigdeli, 2009; Park, 2016; Delimatsis, 2016; Dobson, 2018).

\subsection{Los principios sobre no discriminación entre productos similares}

Los principios sobre no discriminación contenidos en el vigente Acuerdo General sobre Aranceles Aduaneros y Comercio (GATT) de 1994 (el principio del trato general de la nación más favorecida, en su art. I, y el principio del trato nacional en materia de tributación y reglamentación interiores, en su art. III) prohíben a los Miembros de la OMC, con carácter general y sucintamente, que discriminen entre productos similares de distintos Miembros o entre productos similares importados y domésticos.

Esta noción de productos similares se ha interpretado, tradicionalmente, como productos idénticos o con características muy parecidas, atendiendo a varios criterios, 
como la naturaleza y las características físicas de los productos, sus usos finales en el mercado, los gustos y hábitos de los consumidores y las clasificaciones internacionales de los productos a fines arancelarios ${ }^{3}$. Según la jurisprudencia del antiguo sistema del GATT de 1947 y de la actual OMC, el análisis de la similitud entre dos productos se ha centrado, principalmente, en la naturaleza y las características físicas del producto final, tal como llega a la frontera o aduana. Así, no se han venido teniendo en cuenta los diversos procesos o métodos de producción (en inglés "processes and production methods", PPMs) que no dejan una traza física en el producto final (en inglés "nonproduct related PPMs", npr-PPMs). Según este planteamiento, una plancha acero originaria de un país donde se ha producido con pocas emisiones de carbono y una plancha de acero procedente de otro país donde se ha elaborado con cuantiosas emisiones de carbono deberían ser consideradas como productos similares y tratadas igualmente, pues físicamente son idénticas o tienen unas características muy parecidas, siendo irrelevante que tengan distintas huellas de carbono, pues tales huellas son nprPPMs, que no se tienen en cuenta en el análisis de la similitud.

Entre la doctrina se han alzado voces críticas contra esta tradicional interpretación de la noción de productos similares, que corre un tupido velo sobre las condiciones de producción en el país de origen que no se reflejan físicamente en el producto final, defendiendo que en el análisis de la similitud entre dos productos físicamente idénticos o con características muy parecidas se deberían tener mucho más en cuenta otros criterios, como los gustos y hábitos de los consumidores, que sí permitirían distinguir dos productos en función de sus condiciones ambientales de producción y otros npr-PPMs (Howse y Regan, 2000; Pauwelyn, 2007; Globerson, 2013).

Pero otros autores (Jackson, 2000; Low, Marceau y Reinaud, 2011) han venido defendiendo mantener el análisis de la similitud entre productos de los arts. I y III del GATT 1994 basándola en sus aspectos físicos, pues abrirlo en abstracto a la consideración de cualesquiera npr-PPMs introduciría una gran inseguridad jurídica. Así, consideran que sería difícil establecer un límite sobre los posibles tipos de npr-PPMs a tener en cuenta y se podría dar lugar a indebidas injerencias extraterritoriales de un país en las más diversas cuestiones internas de otros países, aunque no repercutiesen físicamente en los productos a importar.

\footnotetext{
3 Véase el informe del Grupo de Trabajo sobre ajustes fiscales en frontera, Instrumentos Básicos y Documentos Diversos (IBDD) 18S/106, 1970, párr. 18. Véase también Choi, 2003.
} 
El informe distribuido por el Órgano de Apelación el 22 de mayo de 2014 sobre el asunto Comunidades Europeas - Productos derivados de las focas, ha confirmado este planteamiento tradicional, entendiendo que los productos derivados de focas capturadas por inuit o por otros cazadores son productos similares, como también son similares los productos derivados de focas cazadas para la gestión sostenible de recursos marinos y las cazadas para otros fines, pues ni la identidad del cazador ni la finalidad de la caza son elementos que diferencien a los productos en cuanto tales (OMC, 2014: párrs. 5.95, 5.117 y 5.130).

Sin embargo, ello no implica que los npr-PPMs nunca puedan ser tenidos en cuenta bajo los acuerdos de la OMC. Se podrá atender a los mismos, pero en el 'lugar' jurídico adecuado. Tanto Jackson (2000: 306 y 307) como Low, Marceau y Reinaud (2011: 7) estiman que la 'sede' apropiada para justificar eventuales distinciones regulatorias entre productos basadas en npr-PPMs no está en el análisis de la similitud entre productos, sino en las excepciones previstas en el propio GATT de 1994, que contemplan determinados objetivos legítimos reconocidos multilateralmente que, bajo ciertas condiciones, pueden justificar la imposición de restricciones al comercio o distinciones entre productos similares basadas en ciertos npr-PPMs.

Por tanto, que una medida basada en npr-PPMs sea incompatible con los principios básicos sobre no discriminación entre productos similares no quita que, finalmente, pueda considerarse justificada bajo alguna excepción, como se viene analizando entre la doctrina (Charnovitz, 2002; Gaines, 2002; Conrad, 2011; Young, 2014; Cooreman, 2017; Maggio, 2017; Sifonios, 2018).

En suma y prima facie, un impuesto o mecanismo de ajuste sobre el carbono en frontera, que grave de manera distinta productos físicamente idénticos o con características muy parecidas, en función de su respectiva huella de carbono, sería considerado incompatible con los principios más básicos del GATT de 1994, pero podría acabar estando justificado bajo alguna de las excepciones contempladas en el mismo.

\subsection{La excepción general sobre medidas relativas a la conservación de recursos naturales agotables}

Al tratar de justificar un impuesto o mecanismo de ajuste sobre el carbono en frontera bajo las excepciones del GATT de 1994, entra en juego su art. XX, titulado 
"Excepciones generales". Dicho precepto reconoce que hay diversos fines legítimos de carácter no comercial, explicitados en una serie de apartados listados, que pueden justificar la imposición de restricciones al comercio o de ciertas distinciones entre productos similares, tendiendo puentes entre las normas de la OMC y otros intereses sociales.

Ninguna de las excepciones recogidas en el texto del art. XX del GATT de 1994, cuya redacción procede del originario GATT de 1947, se refiere explícitamente a medidas relativas o necesarias para luchar contra el cambio climático o para la protección del medio ambiente, pero algunos de sus apartados sí pueden dar cabida a ciertas medidas con una finalidad ambiental.

Una primera alternativa para tratar de justificar un impuesto o mecanismo de ajuste sobre el carbono en frontera pasaría por invocar el apartado b) del art. XX del GATT de 1994, sobre medidas "necesarias para proteger la salud y la vida de las personas y de los animales o para preservar los vegetales". Ahora bien, sobre este apartado b) hay cuantiosa jurisprudencia que, tradicionalmente, le ha dado un alcance estrictamente 'territorial', para justificar medidas que un Miembro adopte para proteger la salud o la vida dentro de su propio territorio evitando que se introduzcan en el mismo productos nocivos (como serían productos carcinógenos, como el amianto, o frutas con parásitos), sin cubrir medidas 'extraterritoriales' relativas a perjuicios que se estén causando desde el extranjero, como las emisiones de carbono generadas durante la elaboración de un producto, y que no dejan traza en el producto final que llega a la aduana, que puede ser, en cuanto tal, completamente inocuo (como, por ejemplo, una plancha de acero) ${ }^{4}$.

Otra alternativa, más viable, es el apartado g) del art. XX del GATT de 1994, sobre medidas "relativas a la conservación de recursos naturales agotables, a condición de que tales medidas se apliquen conjuntamente con restricciones a la producción o al consumo nacionales". Aunque, de entrada, la noción "recursos naturales agotables" parecía pensada para recursos naturales no renovables (como los minerales), la jurisprudencia ha venido haciendo una interpretación evolutiva de esta noción, cubriendo también recursos naturales renovables que pueden desaparecer, como especies de animales o vegetales en peligro de extinción, o deteriorarse, como el "aire puro" (OMC, 1996: párr. 6.37).

\footnotetext{
${ }^{4}$ En tal sentido, Condon (2004) constata que la jurisprudencia avala que el art. XX del GATT de 1994 cubre bajo su apartado b) cuestiones domésticas y no transfronterizas.
} 
Además, como este apartado g) requiere explícitamente que las medidas para la "conservación" se apliquen "conjuntamente con restricciones a la producción o al consumo nacionales", se entiende que este apartado sí puede dar cobertura a medidas con un alcance 'extraterritorial', destinadas a conservar recursos naturales agotables no sólo dentro del territorio del Miembro que adopta las medidas, sino también más allá de su jurisdicción.

Así, el citado apartado g) ya ha servido para justificar, en la práctica del sistema de solución de diferencias de la OMC, restricciones o distinciones entre productos físicamente similares o idénticos en función de ciertos npr-PPMs. Es el caso del emblemático asunto Estados Unidos - Prohibición de las importaciones de camarones y de determinados productos del camarón, donde, en la reclamación presentada por Malasia en virtud del art. 21.5 del Entendimiento sobre Solución de Diferencias (ESD) de la OMC, se acabó reconociendo que los Estados Unidos podían prohibir la importación de camarones procedentes de Malasia mientras dicho país asiático permitiese la pesca de camarones con métodos que causan la muerte accidental de tortugas marinas, que son especies en peligro de extinción. Ciertamente, los camarones capturados en Malasia y los capturados en otros países son productos físicamente similares o incluso idénticos, pues que en su pesca se cause o no la muerte accidental de tortugas marinas es un npr-PPM irrelevante para el análisis de la similitud, pero el apartado g) ampara a un Miembro que, como los Estados Unidos, restrinja las importaciones de camarones basándose en que su método de captura en el país de origen no es respetuoso con el medio ambiente, en este caso con la preservación de las tortugas marinas. La consideración por los Estados Unidos sobre los métodos de pesca usados en aguas del país de origen, más allá de la jurisdicción estadounidense, se estimó que no era una indebida injerencia extraterritorial atendiendo a que las tortugas marinas son una especie altamente migratoria, de modo que los Estados Unidos estaban legitimados para restringir las importaciones de ciertos productos (en sí mismos, totalmente inocuos) en función de un problema o daño ambiental que, aunque tenía su origen en aguas de Malasia, tenía efectos transfronterizos (OMC, 2001: párr. $124)^{5}$.

Cumple advertir que los órganos con funciones jurisdiccionales de la OMC

\footnotetext{
${ }^{5}$ Sobre la jurisprudencia de la OMC relativa al apartado g) del art. XX del GATT de 1994 y las tensiones entre comercio y medio ambiente hay una extensa bibliografía. Entre la doctrina española cabe referirse, inter alia, a Martínez Pérez (1999), Juste Ruiz (2003), Elizalde Carranza (2004), Fajardo del Castillo (2005) y Fernández Egea (2008).
} 
todavía no han resuelto ninguna disputa relacionada específicamente con medidas que establezcan restricciones o distinciones entre productos físicamente similares (o incluso idénticos) en función de su respectiva huella de carbono, pero la indicada jurisprudencia da argumentos para entender que podrían quedar amparadas bajo el apartado g) del art. XX del GATT de 1994, pues la huella de carbono se refiere claramente a la preservación del aire puro y el calentamiento global causado por las emisiones de carbono y otros gases de efecto invernadero es, indudablemente, un problema ambiental transfronterizo, que afecta globalmente a todos los países del mundo con independencia del concreto país donde se generen las emisiones.

Ahora bien, hay que tener en cuenta que, para que una medida quede completamente justificada bajo el art. XX del GATT de 1994 no basta con que quede amparada, de entrada, bajo alguno de los diversos apartados de dicho precepto, sino que también se requiere, adicionalmente, que cumpla con las condiciones horizontales previstas en el encabezamiento o "chapeau" de dicho artículo. Tal encabezamiento requiere que las medidas impugnadas no constituyan "un medio de discriminación arbitrario o injustificable entre los países en que prevalezcan las mismas condiciones o una restricción encubierta al comercio internacional”.

En la práctica del sistema de solución de diferencias de la OMC ya existe bastante jurisprudencia sobre el citado encabezamiento, requiriendo que las restricciones o distinciones regulatorias que eventualmente se hagan entre productos similares de distintos países estén bien diseñadas y aplicadas (de forma transparente, respetando el debido proceso, sin imponer cargas innecesarias...) y respondan, efectivamente y de buena fe, a los legítimos objetivos del apartado invocado o sean, cuanto menos, compatibles con los mismos, sin incurrir en discriminaciones arbitrarias o injustificables entre situaciones comparables (Bartels, 2014).

La exigencia de que la medida enjuiciada no incurra en "discriminaciones arbitrarias o injustificadas entre países en los que prevalezcan las mismas condiciones" suscita dudas entre la doctrina con respecto a una medida que, como un posible impuesto o mecanismo de ajuste sobre el carbono en frontera, venga a gravar los productos en función de su respectiva huella de carbono de forma estrictamente igualitaria, sin tener en cuenta el distinto grado de desarrollo de los países de origen de los productos y pese a que, en materia de reducción de emisiones, el régimen internacional sobre el cambio climático se fundamenta en el principio de 
responsabilidades comunes pero diferenciadas (Morosini, 2010; Davidson Ladly, 2012).

\subsection{La previsión sobre medidas de ajuste fiscal en frontera}

Ante las persistentes dudas acerca de si las excepciones generales del art. XX del GATT de 1994 podrían justificar plenamente un impuesto o mecanismo de ajuste sobre el carbono en frontera, entre la doctrina (Pauwelyn, 2012: 51; Gómez, 2013) se suele considerar que la alternativa más factible para tratar de amparar este tipo de medidas bajo las normas de la OMC (y sin tener que invocar sus exigentes excepciones) es diseñar tal impuesto o mecanismo según la específica figura de los ajustes fiscales en frontera, designados en inglés como "border tax adjustments" y contemplados en el art. II:2(a) del GATT de 1994.

Según este precepto, cualquier Miembro de la OMC puede imponer, "en cualquier momento sobre la importación de cualquier producto, [...] una carga equivalente a un impuesto interior aplicado [...] a un producto nacional similar o a una mercancía que haya servido, en todo o en parte, para fabricar el producto importado".

Un ejemplo tradicional de ajuste fiscal en frontera puede referirse al oro. Así, si un país establece un específico impuesto interior a la producción doméstica de oro, cuando llegue oro de importación o productos de importación conteniendo oro (por ejemplo, joyas o relojes) se les podrá imponer en la frontera, además del arancel aduanero ordinario, una carga específica, equivalente a la que grava al oro doméstico, de acuerdo con el indicado principio del trato nacional en materia de tributación interior.

Para diseñar un impuesto sobre el carbono en frontera que encaje bien en la indicada noción de ajuste fiscal en frontera, la UE debería prever un impuesto interior que gravase ciertos productos domésticos (por ejemplo, el acero o el cemento elaborados dentro de la UE) con un impuesto en función del carbono emitido durante su proceso de producción.

Ello puede resultar complejo, pues en la UE se ha venido prefiriendo, hasta ahora, impulsar la reducción o mitigación de las emisiones de carbono y otros gases de efecto invernadero mediante obligaciones para limitarlas en ciertos sectores y según el indicado Régimen de Comercio de Derechos de Emisión, sin recurrir a semejantes figuras impositivas sobre el carbono emitido. En cualquier caso, un mecanismo de ajuste fiscal en frontera siempre requiere, por definición, una equivalencia entre la tributación que se impone a los productos domésticos mediante un impuesto interior y la 
tributación que se impone a los productos importados mediante la carga que se les aplica en frontera. Por consiguiente, se deberá establecer un sistema que, de algún modo, asegure tal equivalencia.

De entrada, cabría plantearse si el actual régimen de la UE sobre derechos de emisión pudiera ser concebido ya como una especie de impuestos interiores o algo análogo a los mismos. Sin embargo, el Tribunal de Justicia de la UE (TJUE), en su Sentencia de 21 de diciembre de 2011 relativa a la integración de las actividades de aviación dentro del Régimen de Comercio de Derechos de Emisión de la UE, descartó que dicho régimen, basado en la asignación de derechos de emisión por sectores de actividad y la posible comercialización de los derechos de emisión no consumidos, pueda ser considerado como un régimen tributario. Según el TJUE, el indicado Régimen de Comercio de Derechos de Emisión de la UE "no pretende crear un ingreso en favor de los poderes públicos, no permite en modo alguno determinar, basándose en una base imponible y en un tipo definidos a priori, el importe que se devengará por tonelada de combustible consumido por todos los vuelos realizados en un año natural", concluyendo que, "por sus propias características, constituye una medida basada en el mercado y no un derecho, gravamen o tasa” (TJUE, 2011: párrs. 143 y147).

Por consiguiente, la existencia del vigente Régimen de Comercio de Derechos de Emisión en la UE no quita que, si ésta quiere imponer un impuesto sobre el carbono como mecanismo de ajuste fiscal en frontera, tendrá que establecer, simultáneamente, unos impuestos interiores al carbono emitido domésticamente. Esta exigencia presupone que, tal como prevé el Pacto Verde Europeo, el futuro establecimiento por la UE de un impuesto o mecanismo de ajuste sobre el carbono en frontera ad extra exigirá, paralelamente, que la UE emprenda una profunda reforma de la fiscalidad de la energía ad intra, cuestión que podría generar reacciones en contra dentro de la propia UE y sus Estados miembros por motivos políticos y económicos.

Sin perjuicio de ello, el principal escollo jurídico, a la luz de las normas de la OMC, para establecer un impuesto o mecanismo de ajuste del carbono en frontera radica en que la citada previsión del GATT de 1994 sobre ajustes fiscales en frontera alude a un impuesto aplicado a "un producto [...] o a una mercancía que haya servido, en todo o en parte, para fabricar el producto importado". Tradicionalmente, se ha entendido dicha referencia a la "mercancía" como relativa a un ingrediente o insumo físicamente presente en el producto importado (como el oro contenido en joyas o 
relojes). En cambio, en el caso de un impuesto sobre el carbono en frontera, lo que se gravaría no es el producto en cuanto tal ni una mercancía incorporada físicamente dentro de los ingredientes del producto, sino las emisiones de carbono que se generaron en su proceso de producción en el país de origen, de las que no queda un rastro físico en el producto final (acero, cemento...). Pauwelyn (2012) defiende que el Art. II:2(a) del GATT 1994 puede interpretarse dando cobertura también a impuestos relativos a la huella de carbono de un producto, pues, aunque el carbono emitido no sea un ingrediente físico del producto final, tales emisiones de carbono sí son un artículo o "mercancía" que se utiliza para su fabricación'.

Cabe reconocer que una interpretación puramente literal de los términos "mercancía" utilizada, en todo o en parte, para "fabricar el producto importado" difícilmente podría cubrir las emisiones de carbono generadas durante el proceso de elaboración de un producto. Ahora bien, cumple recordar que, según las normas consuetudinarias para la interpretación de los tratados internacionales, codificadas en la Convención de Viena de 1969 y explícitamente aplicables a la interpretación de los acuerdos de la $\mathrm{OMC}^{7}$, en la interpretación de los tratados también hay que tener en cuenta, junto al sentido corriente de sus términos, su "contexto", su "objeto y fin" y, entre otros elementos, "toda norma pertinente de Derecho internacional aplicable en las relaciones entre las partes"8, como las vinculadas con el desarrollo sostenible, contemplado explícitamente como un objetivo en el Preámbulo del Acuerdo por el que se establece la OMC, y que permiten hacer interpretaciones evolutivas ${ }^{9}$.

\section{Consideraciones finales}

\footnotetext{
${ }^{6}$ Pauwelyn (2012: 27-29) considera que un posible antecedente favorable a su tesis sería el informe del Grupo Especial establecido bajo el sistema del GATT de 1947 sobre el asunto Estados Unidos Impuestos al Petróleo y Ciertas Sustancias Importadas, más conocido con el nombre abreviado Estados Unidos - Superfund. Así, el Grupo Especial concluyó que un impuesto de los Estados Unidos sobre determinadas sustancias químicas y sobre importaciones de productos que utilizaron dichas sustancias como insumos en el proceso de producción podían ser objeto de un ajuste fiscal en frontera (GATT de 1947, 1987: párrs. 5.2.1-5.2.10). Ahora bien, Gómez (2013: 25) pone en duda la relevancia de dicho caso, pues el Grupo Especial "no especificó si dichos insumos tenían que ser incorporados al producto final” o podían no serlo.

${ }^{7}$ Según el art. 3.2 del ESD de la OMC, los acuerdos abarcados por ésta deben ser interpretados "de conformidad con las normas usuales de interpretación del Derecho internacional público".

${ }^{8}$ A tenor del art. 31 de la Convención de Viena de 1969 sobre el Derecho de los Tratados.

${ }^{9}$ Sobre la interpretación de los acuerdos de la OMC hay cuantiosa bibliografía. Baste citar aquí, como muestra, a Pauwelyn (2003), Van Damme (2009), Cook (2015) y Qureshi (2015). La interpretación evolutiva acuerdos de la OMC ha sido recientemente analizada por Marceau (2018). En general, sobre la interpretación evolutiva del Derecho internacional, véase el libro Abi-Saab et al. (2019), que incluye una Parte IV con varios capítulos dedicados específicamente a la interpretación evolutiva del Derecho de la OMC.
} 
La Comisión Europea ha incluido, como una de las más destacadas medidas del Pacto Verde Europeo, la propuesta de establecer un impuesto o mecanismo de ajuste sobre el carbono en frontera. Sin embargo, como se ha visto, persisten las incertidumbres jurídicas sobre cómo diseñar tal medida para que sea compatible con las normas de la OMC. Sus previsiones y jurisprudencia todavía no han clarificado todos los requisitos a cumplir por una medida que, como la propuesta, venga a establecer distinciones en la tributación de productos físicamente similares (o incluso idénticos) en función de su respectiva huella de carbono, teniendo en cuenta las emisiones de carbono generadas fuera de la UE, en los respectos países de origen de los productos.

Ante estas incertidumbres del régimen de la OMC, la UE puede actuar aquí de forma asertiva, diseñando y fundamentando cuidadosamente tal propuesta a la luz de la jurisprudencia la OMC sobre otros tipos de medidas comerciales relativas a npr-PPMs y defendiendo una interpretación evolutiva del GATT de 1994 y, en particular, de sus excepciones generales y de sus específicas previsiones sobre ajustes fiscales en frontera, atenta a las normas internacionales relativas a la lucha contra el cambio climático y la promoción de un desarrollo sostenible.

Una vez que la UE haya establecido el impuesto o mecanismo de ajuste sobre el carbono en frontera no es descartable que otros países, incluyendo grandes potencias, cuestionen tal medida ante el sistema de solución de diferencias de la OMC, alegando que es incompatible con sus principios básicos y que tiene un indebido alcance extraterritorial. Pero, como se ha visto, la UE tendría argumentos sólidos para tratar de defender la compatibilidad de su medida con las normas de la OMC adecuadamente interpretadas.

Ciertamente, lo ideal sería lograr una reforma de las normas de la OMC, que ajustase más claramente el tenor de sus previsiones a los actuales retos ambientales y sociales globales ${ }^{10}$. Se podría explicitar en el texto del GATT de 1994, por ejemplo: el alcance de las excepciones generales para abordar problemas transfronterizos como el calentamiento global; o que los ajustes fiscales en frontera pueden referirse a insumos utilizados durante la elaboración de un determinado producto, aunque, como ocurre con

${ }^{10}$ Tal como propone, por ejemplo, Trachtman (2017b: 309), quien considera que la jurisprudencia y las normas de la OMC deberían ser más flexibles para permitir ampliamente "even-handed and proportionate measures to protect the environment", proponiendo incluso un "treaty amendment" en tal sentido. Asimismo, ya hace tiempo que Condon (2006: 276) observa que "many are uncomfortable with the level of ambiguity found in the WTO rules regarding the compatibility of environmentally motivated trade barriers with the WTO" y considera que, aunque un cierto nivel de ambigüedad puede facilitar interpretaciones evolutivas, interesaría clarificar la cuestión textualmente. 
las emisiones de carbono generadas durante el proceso de producción en origen, no dejen una traza física en el producto final.

La Comisión Europea, en un "concept paper" de 2018 sobre la reforma de la OMC, ha defendido las bases institucionales y jurídicas del sistema multilateral del comercio, pero también ha señalado la necesidad de reformarlo profundamente y modernizarlo, subrayando, entre otros aspectos, que éste debería promover un comercio internacional más sostenible y justo. Así, el indicado "concept paper" dedica un específico apartado a la posible contribución de la OMC a "the sustainability objectives of the global community", sosteniendo que la OMC y sus normas deberían enfocarse en mayor medida a la consecución de los ODS, yendo mucho más allá de las actuales negociaciones de la Ronda de Doha sobre prohibición de determinadas formas de subvenciones a la pesca que contribuyen a la sobrecapacidad y la sobrepesca, cuestión contemplada en el ODS 14.6 (Comisión Europea, 2018: 6, sección I.C).

La reforma del régimen de la OMC podría seguir el modelo de los acuerdos comerciales bilaterales o regionales que últimamente está impulsando la UE con terceros países, incorporando previsiones o incluso un nuevo acuerdo multilateral sobre comercio y desarrollo sostenible, que introdujese más referencias sociales y ambientales. Con ello, el régimen de la OMC se actualizaría, estaría menos especializado en la liberalización del comercio y sería más holístico, estableciendo explícitas interconexiones entre las normas comerciales y otros ámbitos del ordenamiento jurídico internacional, como el régimen internacional sobre el cambio climático.

Ahora bien, en el complejo contexto internacional actual, cada vez más multipolar, esa ambiciosa reforma y modernización de la OMC parece tan urgente como difícil de lograr en la práctica. Mientras tanto, es plausible que la UE quiera impulsar, autónomamente, medidas 'correctivas' del comercio internacional, como la comentada propuesta de un impuesto o mecanismo de ajuste sobre el carbono en frontera. 


\section{Referencias bibliográficas}

Abi-Saab, G. et al., eds. (2019). Evolutionary Interpretation and International Law. London: Hart Publishing.

Álvarez Gallego, S. (2015). La huella del carbono de los productos. Madrid: Asociación Española de Normalización y Certificación (AENOR).

Arce González, G. (2014). "Fuga de carbono, hipótesis de refugio de emisiones e hipótesis alternativas. Una revisión de la literatura”. Información Comercial Española, n. ${ }^{\circ} 881,2014$, pp. 167-178.

Babiker, M.H. (2005). "Climate change policy, market structure, and carbon leakage". Journal of International Economic, vol. 65-2, pp. 421-445.

Bartels, L. (2014). "The Chapeau of Article XX GATT: A New Interpretation". University of Cambridge Legal Studies Research Paper Series, Paper 40/2014, pp. 1-19.

Branger, F. y Quirion, P. (2014). "Climate policy and the 'carbon haven'effect". Wiley Interdisciplinary Reviews: Climate Change, vol. 5(1), pp. 53-71.

Charnovitz, S. (2002). "The Law of Environmental 'PPMs' in the WTO: Debunking the Myth of Illegality". Yale Journal of International Law, vol. 27(2), pp. 59-110.

Choi, W.M. (2003). 'Like Products' in International Trade Law: Towards a Consistent GATT/WTO Jurisprudence. Oxford: Oxford University Press.

Comisión Europea (2018). "EU concept paper on WTO reform". Disponible en: https://trade.ec.europa.eu/doclib/docs/2018/september/tradoc_157331.pdf (último acceso 1 de febrero de 2020).

Comisión Europea (2019). "El Pacto Verde Europeo", COM(2019) 640 final, 11 de diciembre de 2019.

Condon, B.J. (2004). "GATT Article XX and Proximity of Interest: Determining the Subject Matter of Paragraphs b and g”. UCLA Journal of International Law and Foreign Affairs, vol. 9(2), pp. 137-162.

Condon, B.J. (2006). Environmental Sovereignty and the WTO - Trade Sanctions and International Law. Ardsley: Transnational Publishers. 
Condon, B. (2009). "Climate Change and Unresolved issues in WTO Law". Journal of International Economic Law, vol. 12(4), pp. 895-926.

Conrad, C.R. (2011), Processes and Production Methods (PPMs) in WTO Law: Interfacing Trade and Social Goals. Cambridge: Cambridge University Press.

Cook, G. (2015). A Digest of WTO Jurisprudence on Public International Law Concepts and Principles. Cambridge: Cambridge University Press.

Cooreman, B. (2017). Global Environmental Protection through Trade: A Systematic Approach to Extraterritoriality. Chelthenham: Edward Elgar.

Cottier, T., Nartova, O., y Bigdeli, S.Z., ed. (2009). International Trade Regulation and the Mitigation of Climate Change, Cambridge: Cambridge University Press.

Davidson Ladly, S. (2012). "Border carbon adjustments, WTO-law and the principle of common but differentiated responsibilities". International Environmental Agreements, vol. 12, pp. 63-84.

Delimatsis, P., ed. (2016). Research Handbook on Climate Change and Trade Law. Cheltenham: Edward Elgar.

Dobson, N.L. (2018). "The EU's conditioning of the 'extraterritorial' carbon footprint: A call for an integrated approach in trade law discourse". Review of European, Comparative and International Environmental Law, vol. 27(1), pp. 75-89.

Douma, W.T. (2017). "The Promotion of Sustainable Development through EU Trade Instruments". European Business Law Review, vol. 28(2), pp. 197-216.

Eliste P. y Fredriksson P.G. (2004). "Does Trade Liberalization Cause a Race-to-theBottom in Environmental Policies? A Spatial Econometric Analysis". En L. Anselin, R.J. Florax y S.J. Rey, S.J., eds., Advances in Spatial Econometrics. Advances in Spatial Science. Berlin - Heidelberg: Springer, pp. 383 y ss.

Elizalde Carranza, M.A. (2004). "La legalidad de las medidas comerciales unilaterales para proteger el medio ambiente en el sistema multilateral del comercio". Revista Interdisciplinar de Gestión Ambiental, año 5, núm. 67, pp. 29-42.

Elliott, J. et al. (2010). "Trade and carbon taxes". American Economic Review, vol. 100(2), pp. 465-469. 
Fajardo del Castillo, T. (2005). "El sistema de solución de controversias de la OMC y el medio ambiente". En J.M. de Faramiñán Gilbert, coord., Globalización y comercio internacional - Actas de las XX Jornadas de la AEPDIRI - Jaén 2003, Madrid: BOE - AEPDIRI - Universidad de Jaén, pp. 131-145.

Fernández Egea, R.M. (2008). Comercio de mercancías y protección del medio ambiente en la OMC, Madrid: Marcial Pons.

Fernández Pons, X. (2015) "Nueva agenda (y promesas) en la Cumbre sobre el Desarrollo Sostenible", Huffington Post, 4 de octubre de 2015. Disponible en: https://www.huffingtonpost.es/xavier-fernandez-pons/nueva-agenda-ypromesas-e b_8232682.html (último acceso 1 de febrero de 2020).

Gaines, S.E (2002). "Processes and Production Methods: How to Produce Sound Policy for Environmental PPM-Based Trade Measures". Columbia Journal on Environmental Law, vol. 27, pp. 383 y ss.

García, R. y Freire, F. (2014). "Carbon footprint of particleboard: a comparison between ISO/TS 14067, GHG Protocol, PAS 2050 and Climate Declaration”. Journal of Cleaner Production, vol. 66, pp. 199-209.

GATT de 1947 (1987). Estados Unidos - Impuestos al Petróleo y Ciertas Sustancias Importadas, Informe del Grupo Especial, L/6175 - 34S/136, adoptado el 17 de junio de 1987.

Gómez, M.F. (2013). “Cambio climático y ajustes fiscales en frontera: análisis jurídico y viabilidad institucional”. Revista de Derecho Económico Internacional, vol. 4(1), pp. 9-41.

Globerson, M. (2013). "Using Border Trade Adjustments to Address Labor Rights Concerns Under the WTO”. Labor and Employment Law Forum, vol. 3(1), pp. 48-87.

Gros, D. y Egenhofer, C. (2011). "The case for taxing carbon at the border". Climate Policy, vol. 11(5), pp. 1262-1268.

Gruni, G. (2018). “Towards a Sustainable World Trade Law? The Commercial Policy of the European Union After Opinion 2/15 CJEU". Global Trade and Customs Journal, vol. 13(1), pp. 4-12. 
Hadjiyianni, I. (2019). The EU as a Global Regulator for Environmental Protection: A Legitimacy Perspective. Oxford: Hart Publishing.

Holzer, K. (2014). Carbon-related Border Adjustment and WTO Law. Cheltenham: Edward Elgar.

Howse, R. y Regan, D. (2000). “The Product/Process Distinction - An Illusory Basis for Disciplining "Unilateralism" in Trade Policy". European Journal of International Law, vol. 11, pp. 249 y ss.

IPPC (2018). Global Warming of $1.5^{\circ}$ C. An IPCC Special Report on the impacts of global warming of $1.5^{\circ} \mathrm{C}$ above pre-industrial levels and related global greenhouse gas emission pathways, in the context of strengthening the global response to the threat of climate change, sustainable development, and efforts to eradicate poverty. Disponible en https://www.ipcc.ch/sr15/ (último acceso 1 de febrero de 2020).

Jackson, J.H. (2000). "Comments on Shrimp/Turtle and the Product/Process Distinction”. European Journal of International Law, vol. 11(2), pp. 303-307.

Juste Ruiz, J. (2003). "Protección del medio ambiente y comercio internacional". Cursos Euromediterráneos Bancaja de Derecho Internacional, vol. 7, pp. 341456.

Kaufmann, C. y Weber, R. H. (2011). “Carbon-related border tax adjustment: mitigating climate change or restricting international trade?". World Trade Review, vol. 10(4), pp. 497-525.

Krenek, A. (2020). "How to implement a WTO-compatible full border carbon adjustment as an important part of the European Green Deal". Österreichische Gesellschaft für Europapolitik (ÖGfE) Policy Brief, 17 January 2020, pp. 1-9. Disponible en: https://oegfe.at/2020/01/wto-compatible-bca-green-deal/ (último acceso 1 de febrero de 2020).

Kuik, O. y Gerlagh, R. (2003). “Trade liberalization and carbon leakage”. The Energy Journal, vol. 24-3, pp. 97-120.

Lomborg, B. (2015). "A Climate Agreement Powered by Hypocrisy", Project Syndicate. Disponible en: https://www.project- 
syndicate.org/commentary/paris-climate-agreement-hypocrisy-by-bj-rn-

lomborg?barrier=accesspaylog (último acceso 1 de febrero de 2020).

Low, P., Marceau, G. y Reinaud, J. (2011). The Interface Between the Trade and Climate Change Regimes: Scoping the Issues. Geneva: WTO.

Maggio, A.R. (2017). Environmental Policy, Non-Product Related Process and Production Methods and the Law of the World Trade Organization. Cham: Springer.

Marceau, G. (2018). "Evolutive Interpretation by the WTO Adjudicator". Journal of International Economic Law, vol. 21(4), pp. 791-813.

Martínez Pérez, E.J. (1999). “Especies migratorias y el artículo XX del GATT: el asunto Estados Unidos - Prohibición de las importaciones de determinados camarones y productos del camarón”. Anuario de Derecho Internacional, vol. XV, pp. 603-628.

Mehling, M. et al. (2019). "What a European 'carbon border tax' might look like”. Vox Column, 10 December 2019. Disponible en: https://voxeu.org/article/whateuropean-carbon-border-tax-might-look (último acceso 1 de febrero de 2020).

Metcalf, G.E. y Weisbach, D. (2009). "The design of a carbon tax". Harvard Environmental Law Review, vol. 33, pp. 499 y ss.

Morosini, F. (2010). "Trade and Climate Change: Unveiling the Principle of Common But Differentiated Responsabilites from the WTO Agreements". The George Washington International Law Review, vol. 42, pp. 713-748.

OMC (1996). Estados Unidos - Pautas para la gasolina reformulada y convencional, Informe del Grupo Especial, WT/DS2/R, de 29 de enero de 1996.

OMC (2001). Estados Unidos - Prohibición de las importaciones de determinados camarones y productos del camarón-Recurso de Malasia al artículo $21.5 \mathrm{del}$ ESD, Informe del Órgano de Apelación, WT/DS58/AB/RW, de 22 de octubre de 2001.

OMC (2014). Comunidades Europeas - Productos derivados de las focas, Informe del Órgano de Apelación, WT/DS400 /AB/R - WT/DS401/AB/R, de 22 de mayo de 2014. 
Park, D.Y., ed. (2016). Legal Issues on Climate Change and International Trade Law. Cham: Springer.

Pauwelyn, J. (2003). Conflicts of Norms in Public International Law - How WTO Law Relates to other Rules of International Law. Cambridge: Cambridge University Press.

Pauwelyn, J. (2007). "U.S. Federal Climate Policy and Competitiveness Concerns: The Limits and Options of International Trade Law". Working Paper - Nicholas Institute for Environmental Policy Solutions, pp. 28-32. Disponible en:

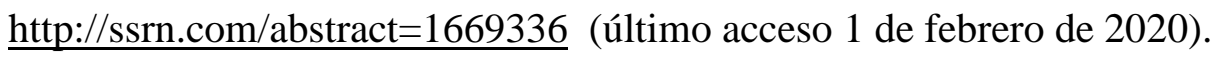

Pauwelyn, J. (2012). “Carbon Leakage Measures and Border Tax Adjustments Under WTO Law". Social Science Research Network, pp. 1-52. Disponible en: https://ssrn.com/abstract=2026879 (último acceso 1 de febrero de 2020).

Pauwelyn, J. (2013). “Chapter 15: Carbon leakage measures and border tax adjustments under WTO law”. En G. Van Calster y D. Prévost, eds., Research Handbook on Environment, Health and the WTO. Cheltenham: Edward Elgar, pp. 448506.

Peters, G.P. (2010). "Policy Update: Managing carbon leakage”. Carbon Management, vol. 1(1), pp. 35-37.

Pirlot, A. (2017). Environmental Border Tax Adjustments and International TradeFostering Environmental Protection. Cheltenham: Edward Elgar.

Porterfield, M.C. (2019). "Border Adjustments for Carbon Taxes, PPMs, and the WTO”. University of Pennsylvania Journal of International Law, vol. 41(4), pp. $1-41$.

Puig Marcó, R. (2020). “La hoja de ruta legislativa del Pacto Verde Europeo”. Terraqui - Actualidad y opinión jurídica del derecho ambiental, 14 de enero de 2020. Disponible en: https://www.terraqui.com/blog/actualidad/la-hoja-de-rutalegislativa-del-pacto-verde-europeo/ (último acceso 1 de febrero de 2020).

Qureshi, A.H. (2015). Interpreting WTO Agreements: Problems and Perspectives, 2nd edition. Cambridge: Cambridge University Press. 
Read, R. (2005). "Process and Production Methods and the Regulation of International Trade". En N. Perdikis y R. Read, eds., The WTO and the Regulation of International Trade: Recent Trade Disputes Between the European Union and the United States, Camberley: Edward Elgar, pp. 239-267.

Sindico, F. (2008). "The EU and Carbon Leakage: How to Reconcile Border Adjustments with the WTO?". European Energy and Environmental Law Review, vol. 17(6), pp. 328-340.

Sifonios, D. (2018). Environmental Process and Production Methods (PPMs) in WTO Law. Cham: Springer.

TJUE (2011). Sentencia de 21 de diciembre de 2011, asunto C-366/10, The Air Transport Association of America et al. y The Secretary of State for Energy and Climate Change, ECLI:EU:C:2011:864.

Trachtman, J.P. (2017a). "WTO Law Constraints on Border Tax Adjustment and Tax Credit Mechanisms to Reduce the Competitive Effects of Carbon Taxes". National Tax Journal, vol. 70(2), pp. 469-494.

Trachtman, J.P. (2017b). "WTO Trade and Environment Jurisprudence: Avoiding Environmental Catastrophe". Harvard International Law Journal, vol. 58(2), pp. 273-309.

Van Damme, I. (2009). Treaty Interpretation by the WTO Appellate Body, Oxford: Oxford University Press.

Veel, P.E. (2009). "Carbon Tariffs and the WTO: An Evaluation of Feasible Policies". Journal of International Economic Law, vol. 12(3), pp. 749-800.

Von der Leyen, U. (2019). “Orientaciones Políticas para la próxima Comisión Europea 2019-2024”. Disponible en: https://ec.europa.eu/commission/sites/betapolitical/files/political-guidelines-next-commission_es.pdf (último acceso $1 \mathrm{de}$ febrero de 2020).

Will, U. (2019). Climate Border Adjustments and WTO Law - Extending the EU Emissions Trading System to Imported Goods and Services. Boston - Leiden: Brill - Nijhoff. 
Woods, N.D. (2006). "Interstate Competition and Environmental Regulation: A Test of the Race-to-the-Bottom Thesis”. Social Science Quarterly, vol. 87(1), pp. 174189.

Wouters, J. et al., eds. (2015). Global Governance through Trade - EU Policies and Approaches. Cheltenham: Edward Elgar.

Wu, P., Xia, B. y Wang, X. (2015). "The contribution of ISO 14067 to the evolution of global greenhouse gas standard - A review". Renewable and Sustainable Energy Reviews, vol. 47, pp. 142-150.

Young, M.A. (2014). “Trade Measures to Address Environmental Concerns in Faraway Places: Jurisdictional Issues". Review of European, Comparative and International Environmental Law, vol. 23(3), pp. 302-317. 